\title{
Declines in Drp1 and Parkin Expression Underlie DNA Damage-Induced Changes in Mitochondrial Length and Neuronal Death
}

\author{
David B. Wang, ${ }^{1}$ Gwenn A. Garden, ${ }^{2}$ Chizuru Kinoshita, ${ }^{1}$ Cody Wyles, ${ }^{1}$ Nasim Babazadeh, ${ }^{1}$ Bryce Sopher, ${ }^{2}$ \\ Yoshito Kinoshita, ${ }^{1}$ and Richard S. Morrison ${ }^{1}$ \\ ${ }^{1}$ Department of Neurological Surgery and ${ }^{2}$ Department of Neurology, University of Washington School of Medicine, Seattle, Washington $98195-6470$
}

\begin{abstract}
Maintaining proper mitochondrial length is essential for normal mitochondrial function in neurons. Mitochondrial fragmentation has been associated with neuronal cell death caused by a variety of experimental toxic stressors. Despite the fact that oxidative stress is a hallmark of neurodegenerative conditions and aging and the resulting activation of p53 is believed to contribute to the neuropathology, little is still known regarding changes in mitochondrial morphology in p53-dependent neuronal death. Therefore, we specifically addressed the relationship between genotoxic stress, p53 activation, and the regulation of mitochondrial morphology in neurons. In cultured postnatal mouse cortical neurons, treatment with the DNA-damaging agent camptothecin (CPT) resulted in elongated mitochondria, in contrast to fragmented mitochondria observed upon staurosporine and glutamate treatment. In fibroblasts, however, CPT resulted in fragmented mitochondria. CPT treatment in neurons suppressed expression of the mitochondrial fission protein Drp 1 and the E3 ubiquitin ligase parkin. The presence of elongated mitochondria and the declines in Drp1 and parkin expression occurred before the commitment point for apoptosis. The CPT-induced changes in Drp1 and parkin were not observed in p53-deficient neurons, while p53 overexpression alone was sufficient to reduce the expression of the two proteins. Elevating Drp1 or parkin expression before CPT treatment enhanced neuronal viability and restored a normal pattern of mitochondrial morphology. The present findings demonstrate that genotoxic stress in neurons results in elongated mitochondria in contrast to fission induced by other forms of stress, and p53dependent declines in Drp1 and parkin levels contribute to altered mitochondrial morphology and cell death.
\end{abstract}

\section{Introduction}

Oxidative stress resulting from mitochondrial dysfunction is associated with the pathogenesis of multiple neurodegenerative diseases and aging (Lin and Beal, 2006). One consequence of oxidative stress is the generation of DNA damage with subsequent induction of the p53 tumor suppressor protein (Morrison et al., 2003). Thus, induction of the $\mathrm{p} 53$ protein in neurons has been demonstrated for a diverse array of nervous system diseases including Huntington's disease, Parkinson's disease, and Alzheimer's disease as well as nervous system injury (Morrison et al., 2003; Bae et al., 2005). Numerous lines of evidence suggest that p53 induction is responsible for neuronal damage.

Received July 12, 2012; revised Nov. 27, 2012; accepted Nov. 29, 2012.

Author contributions: D.B.W., G.A.G., B.S., and R.S.M. designed research; D.B.W., G.A.G., C.K., C.W., N.B., B.S.,

Y.K., and R.S.M. performed research; R.S.M. contributed unpublished reagents/analytic tools; D.B.W., G.A.G., B.S., Y.K., and R.S.M. analyzed data; D.B.W., G.A.G., B.S., Y.K., and R.S.M. wrote the paper.

This work was supported by grants from the National Institutes of Health (NS35533 and NS056031) and from the American Heart Association (10GRNT2640173) to R.S.M., and by a National Institute of Neurological Disorders and Stroke Institutional Center Core Grant to support the viral core facility in the Neuroproteomics Center at the University of Washington (NS055088). We acknowledge Dr. Takuma Uo for thoughtful discussions of the data and for generating the mitoDsRed 2 and Drp1 constructs. We also thank Min Spenser and Wee Sze Wan for generation and analysis of TEM images and Chihong Song for assistance with Western blot analysis.

The authors declare no competing financial interests.

Correspondence should be addressed to Dr. Richard S. Morrison, Department of Neurological Surgery, University of Washington School of Medicine, Box 356470, Seattle, WA 98195-6470. E-mail: yael@u.washington.edu.

DOI:10.1523/JNEUROSCI.3365-12.2013

Copyright $\odot 2013$ the authors $\quad 0270-6474 / 13 / 331357-09 \$ 15.00 / 0$
Mitochondria change size and shape by undergoing fission and fusion, processes that are orchestrated by a well conserved cellular machinery comprised of dynamin-related GTPases, dynamin-related protein 1 (Drp1) for fission and mitofusins (Mfn1/2), and optic atrophy-1 for fusion (Chen and Chan, 2005). Recent studies with neurons demonstrate that diverse forms of acute neurotoxic stress commonly cause mitochondrial fission and that enhancing the expression of fusion proteins or suppressing Drp1 activity reduce fission as well as cell death (Barsoum et al., 2006; Wang et al., 2008; Liot et al., 2009). Despite the likely contribution of p53 to neuronal dysfunction and loss due to oxidative stress associated with neurodegenerative conditions and aging, little progress has been made in characterizing how DNA damage directly alters mitochondrial morphology in compromised neurons. The p53-dependent apoptotic pathway mobilizes the pro-apoptotic protein Bax through the transcriptional activation of $\mathrm{BH} 3$ domain-only proteins such as PUMA (Uo et al., 2007).

In addition to its activity in permeabilizing the outer mitochondrial membrane, Bax promotes mitochondrial fragmentation during apoptosis by forming a complex with Drp1 and Mfn2 at fission sites (Karbowski et al., 2002). How Bax is recruited to the mitochondria, however, may dictate its effect on mitochondrial morphology as Bax is also known to function as a fusion protein under physiological conditions (Karbowski et al., 2006). As a transcriptional regulator, p53 may also have a direct influ- 
ence on the expression and function of proteins regulating mitochondrial morphology.

In the present study, we found that DNA damage induced by the topoisomerase I inhibitor, camptothecin (CPT), in cultured cortical neurons does not cause fragmented but rather elongated mitochondria, which is, at least in part, due to decreased expression of Drp1 and parkin. Overexpression of Drp1 or parkin mitigated the mitochondrial elongation and cell death induced by CPT. Staurosporine, a p53-independent apoptotic stress, caused mitochondrial fragmentation as previously reported while CPT treatment of mouse embryonic fibroblasts resulted in mitochondrial fragmentation. Thus, our findings suggest that p53dependent neuronal cell death is uniquely associated with a net increase in mitochondrial length involving reduced expression of Drp1 and parkin. The changes in mitochondrial morphology mediated by p53 could affect mitochondrial function, transport, and, in conjunction with reduced parkin expression, mitophagy in neurons during the course of aging and neurodegenerative disease.

\section{Materials and Methods}

Animals and cell culture. Primary cultures of postnatal cortical from wildtype $\left(\mathrm{p} 53^{+/+}\right)$, p53-deficient $\left(\mathrm{p} 53^{-/-}\right)$, and Bax-deficient $\left(\mathrm{Bax}^{-/-}\right)$ newborn mice (postnatal day 0 ; P0), hippocampal neurons from $\mathrm{P} 0$ $\mathrm{p} 53^{+/+}$mice and cerebellar granule neurons from P7 $\mathrm{p} 53^{+/+}$mice were prepared using either sex as previously described (Xiang et al., 1996). Overall neuronal viability was monitored morphologically based on phase-contrast microscopy (Xiang et al., 1996), nuclear morphology (Hoechst 33258 staining), and EGFP fluorescence of infected neurons. At least three random fields per condition at $32 \times(\sim 30$ neurons per field $)$ were blindly counted for neuronal viability. Mouse embryonic fibroblasts (MEF) were prepared and maintained as previously described (Uo et al., 2007).

Immunoblotting. Protein extracts were prepared in SDS-PAGE sample buffer (10\% glycerol, $62.5 \mathrm{~mm}$ Tris-HCl, pH 6.8, 2\% SDS) and subjected to gel electrophoresis using $10 \mu \mathrm{g}$ of protein/lane on $4-15 \%$ SDS gradient polyacrylamide gels (Bio-Rad) followed by immunoblotting as previously described (Uo et al., 2007). For quantification, images were scanned and measured for pixel intensity using National Institutes of Health (NIH) ImageJ software, and normalized against $\beta$-actin values. Primary antibodies and their dilutions used were as follows: rabbit polyclonal activated caspase-3 (\#9661, 1:1000; Cell Signaling Technology), mouse monoclonal $\beta$-actin (clone AC-15, 1:10000; Sigma), mouse monoclonal parkin (PRK8, 1:500; Santa Cruz Biotechnology), mouse monoclonal Drp1 (clone 8/DLP1, 1:1000; BD Biosciences), and rabbit polyclonal p53 (FL-393, 1:1000; Santa Cruz Biotechnology). Horseradish peroxidase-conjugated secondary antibodies (1: 2000) were from GE Healthcare.

Immunofluorescence. Cells were cultured in Nunc 4-well multidish or on Thermanox plastic coverslips (Nalge Nunc International). Cultured cells were fixed in $2 \%$ paraformaldehyde (PFA) in $0.1 \mathrm{M}$ phosphate buffer, $\mathrm{pH} 7.4$, permeabilized with $0.2 \%$ Triton $\mathrm{X}-100$, and processed for immunostaining as described previously (Xiang et al., 1996). Fluorescent microscopic images were captured on an Axiovert 200 inverted microscope (Carl Zeiss Microimaging) equipped with a cooled CCD camera (SensiCam; Cooke). Images to be directly compared were captured and processed in an identical manner with Slidebook imaging software (Intelligent Imaging Innovations) and Adobe Photoshop (version 7.0.1. or CS2 9.0.2). Primary antibodies used were mouse monoclonal Drp1 clone 8/DLP1 (1:200; BD Biosciences) and chicken Tuj1 (1:500; Aves). Nuclei were labeled with Hoechst $33258(2.5 \mu \mathrm{g} / \mathrm{ml})$. Alexa Fluor dyeconjugated secondary antibodies (1:400) were from Invitrogen. Fluorescent images of mitochondrial-targeted DsRed2 (mitoDsRed2) were subjected to constrained iterative deconvolution using Slidebook.

Plasmid construction and lentivirus production. Production of DNA constructs and lentivirus for FLAG-tagged human Drp1 isoform 1, EGFP, and mitoDsRed2 has been described (Uo et al., 2009). Coexpression of internal ribosomal entry site (IRES)-driven EGFP was used as an infection marker. Human parkin cDNA (\#23935; Addgene) was PCR cloned into the pSico plasmid with IRES-driven NLS-myc. The Drp1 shRNA sequence was purchased from Sigma (Clone ID: NM_152816.1-1101s1c1; Uo et al., 2009). A validated murine parkin shRNA sequence (CGTGATCTGTTTGGACTGTTT) (Kim et al., 2011) driven by the U6 promoter was packaged into the pSico plasmid. Plasmids were packaged into lentiviral vectors as previously described (Uo et al., 2009). All of the PCR-cloned sequences in these vectors were confirmed by DNA sequence analysis. Adenoviral vectors carrying the human 553 gene (p53-Ad) or the $\beta$-galactosidase gene (LacZ-Ad) were obtained and propagated as previously described (Xiang et al., 1996).

Assessment of mitochondrial morphology. The extent of morphological changes in mitochondria visualized with mitoDsRed2 fluorescence was determined as follows. First, we surveyed the lengths of discretely distributed mitochondria in the neurites of nontreated postnatal cortical neurons using the ruler function provided in Slidebook and determined that the majority fell within the range of $2-3 \mu \mathrm{m}$. Neurons were then defined as exhibiting excessive elongation or fragmentation if they displayed multiple $(>5)$ cases of mitochondria longer than $3 \mu \mathrm{m}$ or shorter than 2 $\mu \mathrm{m}$, respectively. Ten images were taken per condition (with a $32 \times$ objective) for three separate experiments. Images were coded and counted by two separate observers blind to the treatments.

Electron microscopic assessment of mitochondrial morphology, number, and size. Cortical neurons were plated on Aclar plastic coverslips and treated at day 3 with CPT or dimethylsulfoxide (DMSO) for $12 \mathrm{~h}$. Cultures were fixed with $2 \%$ PFA and $2.5 \%$ glutaraldehyde in $0.1 \mathrm{~m}$ cacodylate buffer. Fixed cultures were stained with $1 \%$ osmium tetroxide, dehydrated in graded alcohol, and embedded in Epon. Semithin sections were cut and stained with toluidine blue to visualize neuronal structures. Ultrathin sections were then cut from select areas and stained with $2 \%$ uranyl acetate followed by Reynold's lead. For each experimental condition, electron micrographs from regions containing only neurites were obtained using a Philips CM10 transmission electron microscope. The micrographs for each condition were digitized and analyzed by an observer with no knowledge of the experimental conditions. Total neurite area was measured by density segmentation using NIH ImageJ software. The number of mitochondria per unit area was determined after counting all neuritic mitochondria in each micrograph and dividing by the neurite area for that micrograph. Mitochondrial length was measured by placing a tip-to-tip line across the longest axis of each mitochondrion using the straight line tool in NIH ImageJ. The mean mitochondrial length was calculated for each micrograph $(N=95$ for control and 92 for CPT-treated). Results were compared using an unpaired Student's $t$ test in the Prism Software program (GraphPad).

Real-time reverse transcription-PCR. Total RNA was isolated from cultured neurons using an RNeasy isolation kit (Qiagen). Total RNA was reverse transcribed using SuperScriptII reverse transcriptase (Invitrogen) according to the manufacture's instruction. The cDNAs were subjected to real-time reverse transcription (RT)-PCR using commercially available Drp1 primers and probe (Assay ID Mm00679214_g1; Invitrogen) and custom parkin primers (aagaagaccaccaagccttgtc and caaaccagtgatctcccatgc) and probe (agtggtgctggaactgtggct; Invitrogen) selected with the Primer3 program (http://frodo.wi.mit.edu/primer3/). Samples were analyzed on a StepOnePlus Real-Time RT-PCR system (Applied Biosystems) using a $60^{\circ} \mathrm{C}$ annealing temperature and normalized to mouse $18 \mathrm{~S}$ RNA.

\section{Results}

\section{DNA damage does not induce mitochondrial fragmentation in mouse postnatal cortical neurons}

Increased mitochondrial fission or fragmentation is a typical mitochondrial morphological response to apoptosis-inducing stress in both non-neuronal and neuronal cells (Youle and Karbowski, 2005; Barsoum et al., 2006; Jahani-Asl et al., 2007; Benard and Karbowski, 2009). The majority of the apoptotic stresses thus far tested do not involve or depend on the activation of the p53 tumor suppressor protein (Messmer and Brüne, 1996). Since p53 is activated by oxidative stress in numerous models of injury and 

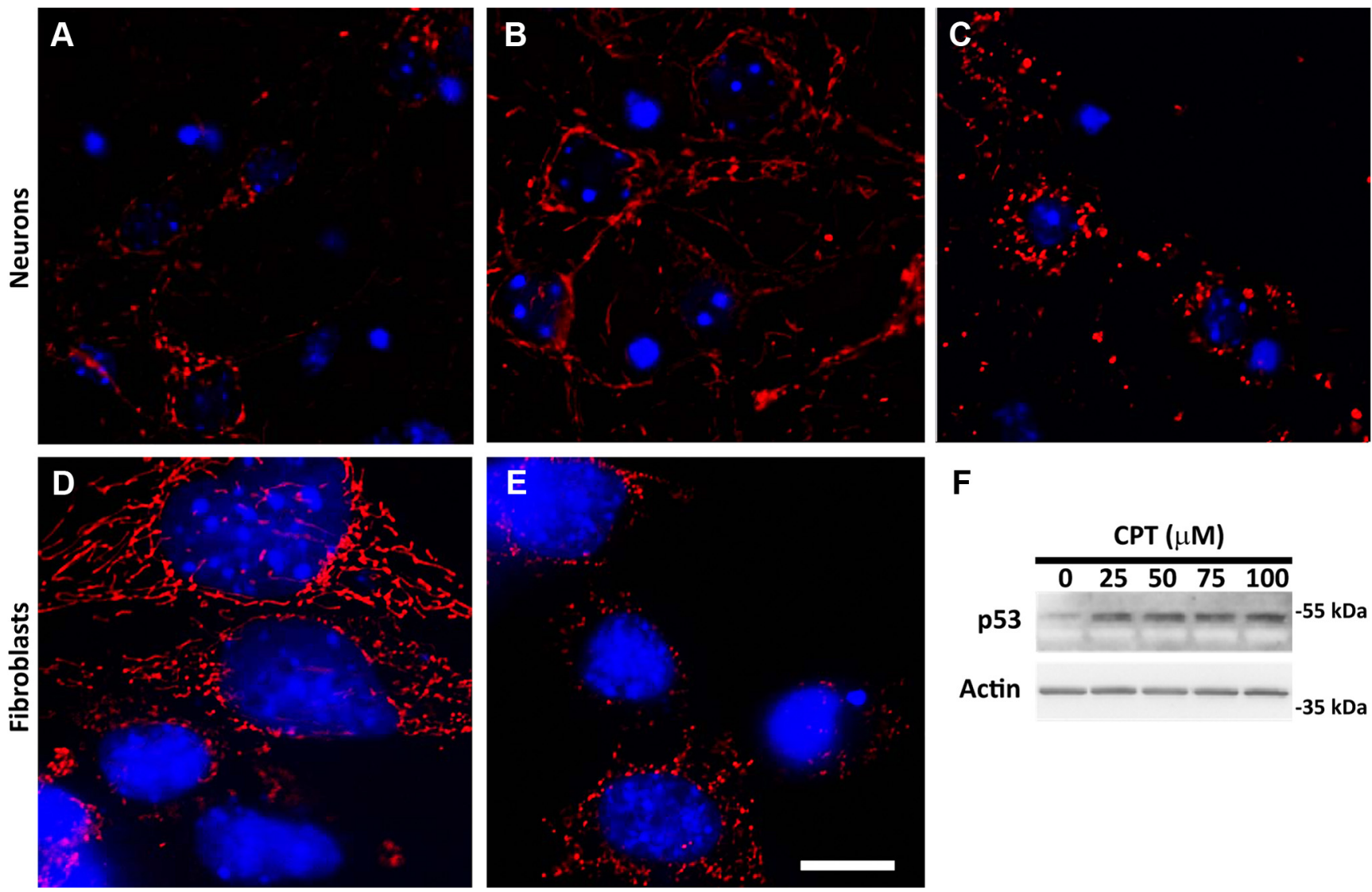

Figure 1. CPT treatment increases mitochondrial length in neurons but induces mitochondrial fragmentation in MEFs in vitro. Postnatal mouse cortical neurons $(\boldsymbol{A}-\boldsymbol{C})$ or MEFs $(\boldsymbol{D}-\boldsymbol{E})$ were plated on Thermanox coverslips and infected with lentivirus expressing mitoDsRed2 (10 MOI) $24 \mathrm{~h}$ after plating. Cells were treated with DMSO (A, D: control), CPT (B, E: $5 \mu \mathrm{m}$ for neurons, $100 \mu \mathrm{m}$ for MEFs), or staurosporine $(C: 0.5 \mu \mathrm{M}) 3 \mathrm{~d}$ after infection and fixed $12 \mathrm{~h}$ after treatment for mitoDsRed 2 fluorescence microscopy to visualize mitochondrial morphology. Note that morphologically discernible cell death becomes apparent only after $20 \mathrm{~h}$ of treatment both for neurons and MEFs under these CPT treatment conditions. Images were taken with a $63 \times 0$ bjective using Slidebook and exported with the $\gamma$ factor set at 0.7 to better represent faint DeRed 2 fluorescence. Neuron cultures were immunostained for $\beta$-tubulin III (TuJ1 antigen), a neuronal marker, to confirm that all the mitoDsRed2 fluorescence shown is associated with neurons (data not shown) while nuclei were visualized by Hoechst 33258 staining (blue). Scale bar, $10 \mu \mathrm{m}$. The images are representative of two to three independent experiments. $F$, MEFs were treated with increasing concentrations of $\mathrm{CPT}$, and $12 \mathrm{~h}$ later protein samples were prepared and analyzed for expression of $\mathrm{p} 53 \mathrm{by}$ Western blot. $\beta$-Actin was used as an internal loading control.
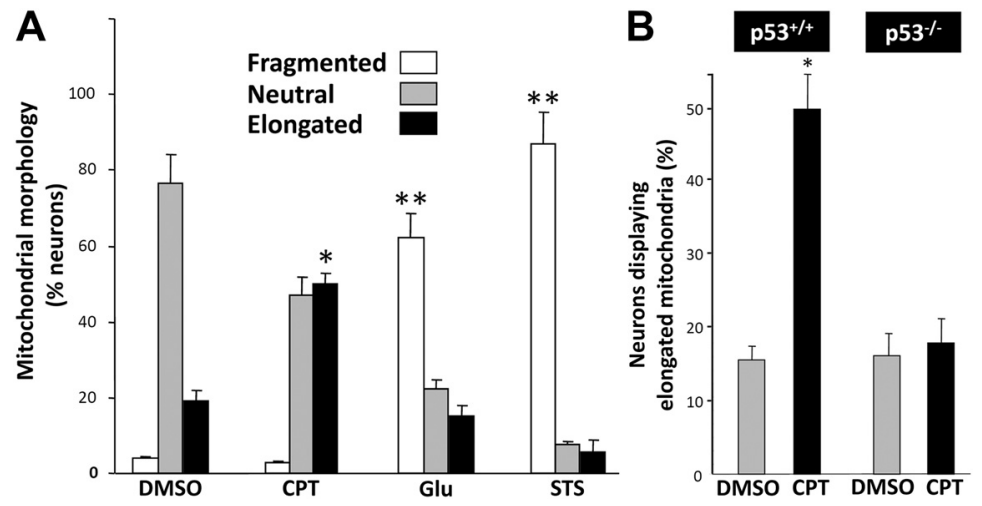

Figure 2. CPT treatment increases mitochondrial length, unlike other neurotoxic stresses, in a p53-dependent manner. $\boldsymbol{A}$, Glutamate (Glu) and staurosporine (STS) increased the percentage of neurons with smaller (fragmented) mitochondria, while (PT increased the percentage of neurons with elongated mitochondria. $\boldsymbol{B}$, In contrast to wild-type $553^{+/+}$neurons, (PT did not elicit a mitochondrial response in $\mathrm{p} 53^{-/-}$neurons. Cortical neurons were infected with lentivirus expressing mitoDsRed2 (10 MOI) $24 \mathrm{~h}$ after plating. Cells were treated with DMSO (control), (PT $(5 \mu \mathrm{m})$, glutamate $(50 \mu \mathrm{m})$, or staurosporine $(0.5 \mu \mathrm{m}) 3 \mathrm{~d}$ after infection and fixed $12 \mathrm{~h}$ after treatment. Mitochondrial length was determined based on the mitoDsRed2 fluorescence as described (see Materials and Methods), while Tuj1 immunostaining was used to identify neurons. Percentage of neurons displaying fragmentation, elongation, or no change (neutral) in mitochondrial morphology was calculated and presented as the mean \pm SEM three independent experiments. ${ }^{*}, *$, Significantly different from all other conditions $(p<0.01$ and $p<0.001$, respectively, one-way ANOVA using Tukey's post hoc test). disease, we sought to determine if mitochondrial morphology is deregulated in a p53-dependent model of apoptosis induced by genotoxic stress via CPT treatment in postnatal cortical neurons in culture.

In DMSO-treated postnatal cortical neurons, mitochondria displayed a short tubular-shaped morphology and were randomly distributed throughout the neurites (Fig. 1A). Using wide field fluorescence imaging, baseline neuritic mitochondrial profiles measured $2-3 \mu \mathrm{m}$. Accordingly, we defined neurons possessing neuritic mitochondrial profiles longer or shorter than this normal range as displaying elongated or fragmented mitochondria, respectively (see Materials and Methods for more detail). When evaluated by this criterion, DMSO-treated control cultures had $10-20 \%$ of neurons displaying elongated mitochondria as a baseline rate and $5-10 \%$ of neurons displaying fragmented mitochondria (Fig. 
A

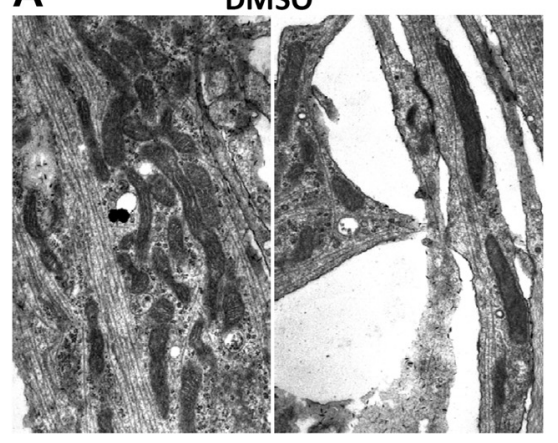

Camptothecin

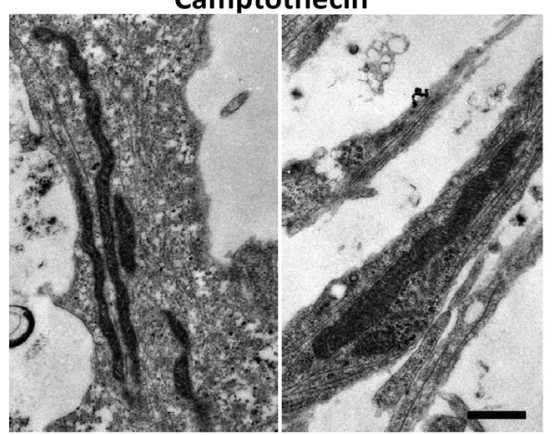

B

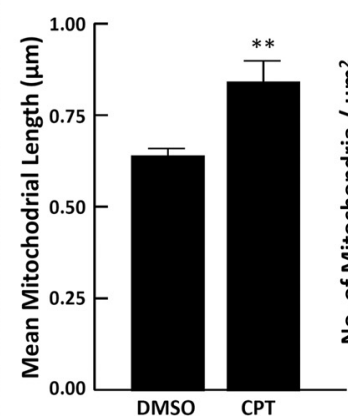

C

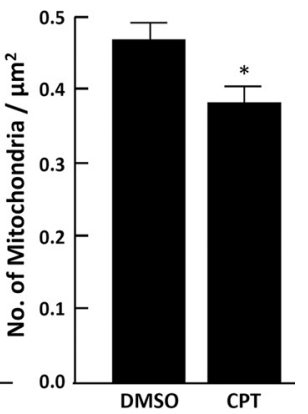

Figure 3. (PT induced increased mitochondrial length and decreased mitochondrial density in neurites as measured by TEM. A, Cortical neurons were treated $3 \mathrm{~d}$ after plating with CPT ( $5 \mu \mathrm{M}$ ) or vehicle (DMSO) and fixed for TEM $12 \mathrm{~h}$ later. TEM images of CPT-treated neurons revealed longer mitochondria in neuronal processes when compared with DMSO-treated neurons. Two representative images are shown for each condition. Scale bar, $1 \mu \mathrm{m}$. Length $(\boldsymbol{B})$ and the number per unit area $\left(\mu \mathrm{m}^{2}\right)(\boldsymbol{C})$ of mitochondria in neurites were calculated from TEM images $(N=95$ for control and 92 for (PT-treated from a single experiment) using unbiased methods and presented as mean \pm SEM. ${ }^{*}$, ${ }^{* *}$, Significantly different from DMSO-treated neurons ( $p<0.05$ and $p<0.01$, respectively; Student's $t$ test).
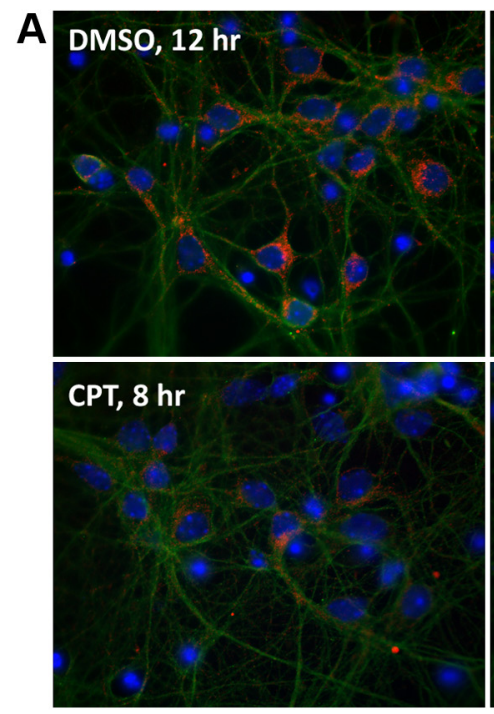
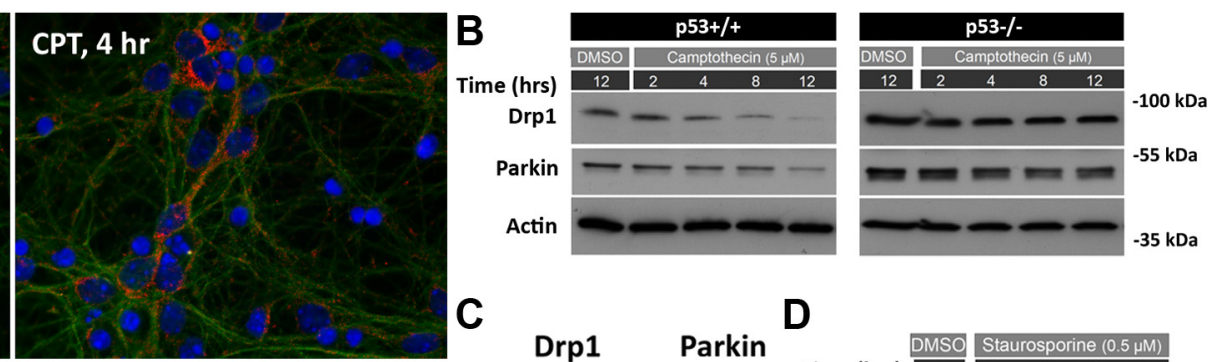

C

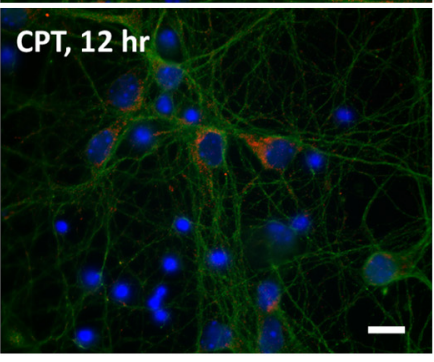

Drp1

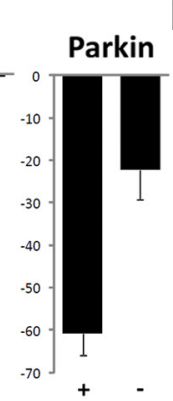

D
$-35 \mathrm{kDa}$
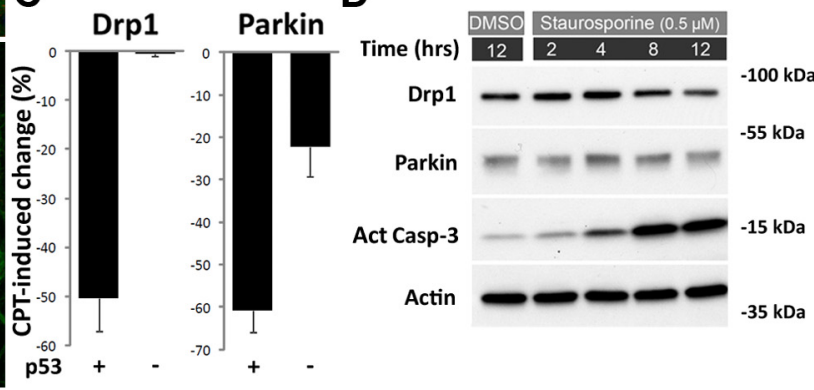

Figure 4. CPT suppresses Drp1 and parkin protein expression in postnatal cortical neurons in a p53-dependent manner. A, p53 ${ }^{+/+}$neurons were treated with DMSO (control, $12 \mathrm{~h}$ only) or CPT $(5 \mu \mathrm{m}) 3 \mathrm{~d}$ after plating for 4, 8, and $12 \mathrm{~h}$, fixed and immunostained for Drp1(red) and Tuj1 (green) with Hoechst 33258 used to delineate nuclei (blue). Scale bar, $10 \mu \mathrm{m}$. The images are representative of four independent experiments. $\boldsymbol{B}, \mathrm{p5}^{+/+}$or $\mathrm{p5} 3^{-/-}$neurons were treated with DMSO (control, $12 \mathrm{~h}$ only) or (PT ( $\left.5 \mu \mathrm{M}\right) 3 \mathrm{~d}$ after plating and $2,4,8$, and $12 \mathrm{~h}$ later protein samples were prepared and analyzed for expression of Drp1 and parkin by Western blot. $\beta$-Actin was used as an internal loading control. C, Western blot results including those shown in $\boldsymbol{B}$ were quantified. In $553^{+/+}$neurons the average CPT-induced decline in Drp1 and parkin expression at $12 \mathrm{~h} \mathrm{relative} \mathrm{to} \mathrm{DMSO} \mathrm{control} \mathrm{(and} \mathrm{normalized} \mathrm{to} \beta$-actin) was $50.53 \pm 6.60 \%$ (mean \pm SEM, $p<$ 0.001 , Student's $t$ test, $N=20)$ and $60.74 \pm 5.32 \%(p<0.001, N=9)$, respectively. CPT did not reduce Drp1 levels in p53 ${ }^{-1-}$ neurons $(0.53 \pm 0.53 \%, N=3)$, but did induce a significant reduction in parkin expression $(22.3 \pm 7.02 \%, p<0.05, N=2)$, although it was substantially less than that observed in CPT-treated $p 53^{+/+}$neurons. $D$, p53 $3^{+/+}$neurons were treated with DMSO (control, $12 \mathrm{~h}$ only) or staurosporine $(0.5 \mu \mathrm{M}) 3 \mathrm{~d}$ after plating and $2,4,8$, and $12 \mathrm{~h}$ later protein samples were prepared and analyzed for expression of Drp1, parkin, and activated caspase-3 by Western blot. $\beta$-Actin was used as an internal loading control. Blots are representative of at least three separate experiments.

$2 A$ ). The mitochondria in neurons treated with glutamate (data not shown) or staurosporine (Fig. 1C) were small and fragmented, with $60 \%$ or $80 \%$ of neurons displaying mitochondrial fragmentation, respectively (Fig. 2A), consistent with previous findings (Young et al., 2010). Mitochondrial fragmentation was also observed with the mitochondrial complex II inhibitor, 3-nitropropionic acid (3-NP; data not shown), as previously reported (Liot et al., 2009). In marked contrast, however, the addition of the DNA-damaging agent CPT induced no sign of mitochondrial fragmentation in postnatal mouse cortical neurons. Rather, mitochondrial structures in neurites appeared longer in CPT-treated neurons (Fig. 1B), suggesting induction of net mitochondrial elongation instead of fragmentation. This was confirmed by quantitative assessment demonstrating that $50 \%$ of neurons displayed elongated neuritic mitochondria in CPT- treated cultures in contrast to $20 \%$ in control (DMSO-treated) cultures (Fig. 2A). Since standard fluorescence microscopy does not have sufficient resolution to distinguish between individual mitochondria and groups of mitochondria in close proximity, we evaluated the effect of CPT treatment on neuritic mitochondria using transmission electron microscopy (TEM). TEM analysis demonstrated that DNA damage significantly increased the average length of individual mitochondria while significantly reducing the number of mitochondria per unit area in the neurites (Fig. 3). However, in MEFs that normally display long networked mitochondria (Fig. 1D), CPT treatment, which activates p53 in human and mouse fibroblasts (Fig. 1F; Tomicic et al., 2005; Wu et al., 2009), resulted in a significant degree of mitochondrial fragmentation and a diminution of mitoDsRed2 fluorescence (Fig. $1 E)$. Thus, contrary to the results from the majority of previous 

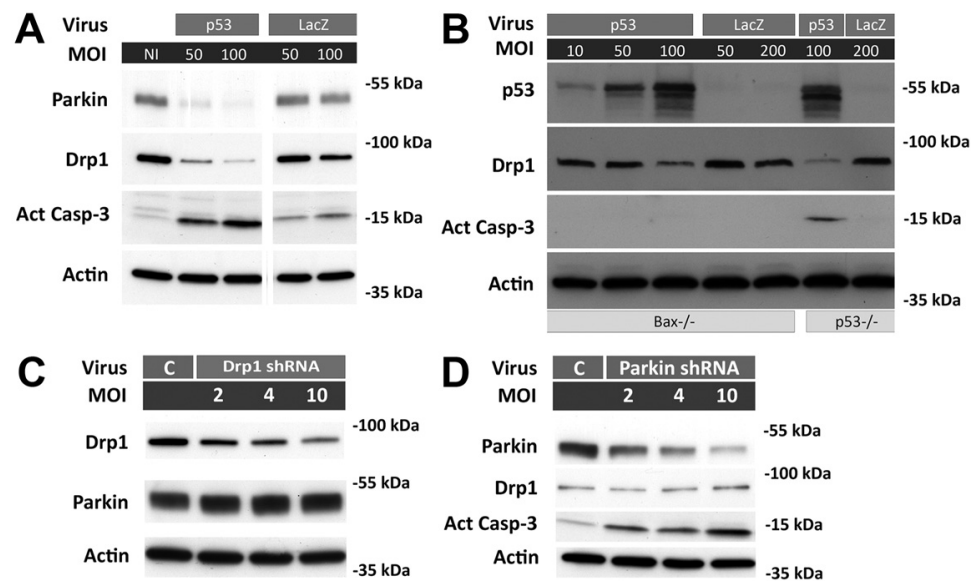

Figure 5. Increased p53 expression is sufficient to induce a decline in parkin and Drp1 contributing to neuronal cell death. $\boldsymbol{A}$, $\mathrm{p} 53^{-1-}$ neurons were infected with adenovirus expressing human $\mathrm{p} 53$ (p53) or $\beta$-galactosidase (LacZ) at varying MOls. Protein samples were prepared $48 \mathrm{~h}$ after infection and analyzed for expression of Drp1, parkin, and activated caspase- 3 by Western blot. $\beta$-Actin was used as an internal loading control. $\boldsymbol{B}$, Bax $^{-1-}$ neurons were infected $1 \mathrm{~d}$ after plating with adenovirus expressing human $p 53$ or $\beta$-galactosidase at varying M0ls. Protein samples were prepared $48 \mathrm{~h}$ after infection and analyzed for expression of $\mathrm{p} 53$, Drp1, and activated caspase-3 by Western blot. $\beta$-Actin was used as an internal loading control. $\boldsymbol{C}, \boldsymbol{D}$, Cortical neurons were infected $1 \mathrm{~d}$ after plating with increasing MOls of lentivirus expressing shRNA against Drp1 or parkin. Protein samples were prepared $3 \mathrm{~d}$ after infection and analyzed for expression of Drp1, parkin, and activated caspase-3 (parkin only). $\beta$-Actin was used as an internal loading control. Two different shRNAs against each protein were tested, with similar results. All blots are representative of at least two separate experiments.

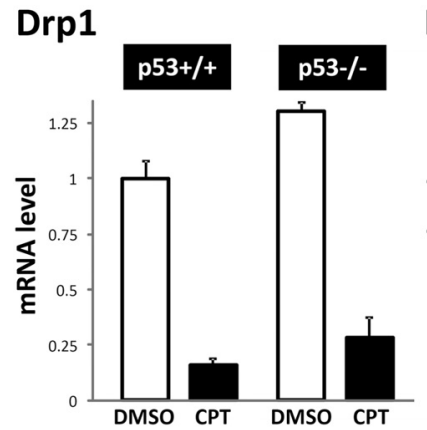

Parkin

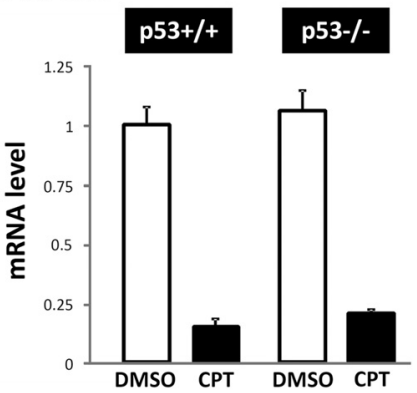

Figure 6. CPT suppresses Drp 1 and parkin mRNA expression. $\mathrm{p} 53^{+/+}$or $\mathrm{p} 53^{-1-}$ neurons were treated with DMSO (control) or CPT ( $5 \mu \mathrm{m}) 3 \mathrm{~d}$ after plating for $12 \mathrm{~h}$. Expression levels of Drp1 and parkin mRNA were analyzed by real-time RT-PCR (see Materials and Methods). The results obtained were first normalized against levels of ribosomal protein S18 (RPS18) mRNA used as an internal control for RNA loading and then presented relative to the levels in DMSOtreated $\mathrm{p} 53^{+/+}$neurons. The data are mean \pm SEM three separate experiments done in duplicates.

studies in neurons and non-neuronal cells using a variety of apoptotic stressors, CPT-induced DNA damage in postnatal cortical neurons did not lead to fragmented mitochondria but to elongated mitochondria. Importantly, in p53-deficient $\left(\mathrm{p} 53^{-1-}\right.$ ) neurons that are resistant to CPT (Xiang et al., 1996, 1998), CPT treatment did not result in elongated mitochondria (Fig. $2 B$ ), implicating DNA damage-induced p53 activation in the observed mitochondrial changes.

\section{CPT treatment reduces Drp1 and parkin expression in a p53-dependent manner}

The longer mitochondria observed following CPT treatment could be a result of decreased fission and/or increased (or sustained) fusion activity. We have already shown that CPT treatment does not appreciably affect the levels of fusion proteins (Mfn1/Mfn2; Uo et al., 2009). In the present study, therefore, we initially focused on the fission protein Drp1. We have reported that shRNA-based suppression of Drp1 results in elongated mitochondria and cell death in cortical neurons (Uo et al., 2009). As seen in Figure 4A, Drp1 immunoreactivity was reduced as early as $4 \mathrm{~h}$ after CPT treatment, relative to DMSO, in postnatal cortical neurons with further decreases seen with increasing time. The reduction in Drp1 immunoreactivity in postnatal cortical neurons was confirmed by Western blot quantification (Fig. $4 B, C$ ). CPT treatment also reduced expression of parkin (Fig. $4 B, C$ ), a protein involved in mitochondrial fusion/ fission (Poole et al., 2008), mitophagy (Dagda et al., 2009), and mitochondrial trafficking (Wang et al., 2011b). Although the decline required $8-12 \mathrm{~h}$ to become noticeable, it occurred well before the initiation of morphological cell death $(>20$ h). Staurosporine treatment, which resulted in fragmented mitochondria (Figs. 1C, 2), did not affect Drp1 or parkin levels despite inducing elevated expression of activated caspase- 3 (Fig. 4D).

The temporal relationship between declines in Drp1 and parkin expression (Fig. $4 B$ ) and p53 induction, the latter occurring as early as $2 \mathrm{~h}$ after CPT treatment (Uo et al., 2007), prompted us to examine whether the changes in Drp1 and parkin levels could occur downstream of p53 induction. In p53 $3^{-1-}$ cortical neurons, CPT treatment did not reduce Drp1, and while CPT induced a statistically significant decrease in parkin in $\mathrm{p} 53^{-1-}$ neurons, it was substantially less than that observed in

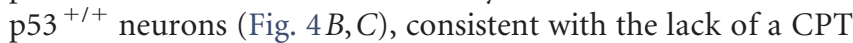
effect on mitochondrial morphology (Fig. 2B). Conversely, adenovirus-mediated overexpression of $\mathrm{p} 53$ in $\mathrm{p}^{+/+}$(data not shown) and $\mathrm{p}^{-1-}$ cortical neurons (Fig. $5 A, B$ ) reduced Drp1 and parkin levels while $\beta$-galactosidase overexpression did not. Overexpression of $\mathrm{p} 53$ in $\mathrm{Bax}^{-1-}$ neurons still reduced Drp1 levels (Fig. 5B), suggesting that CPT-induced decreases in Drp1 are not a result of cell death-associated biochemical changes as these neurons are not killed by p53 activation (Xiang et al., 1998). To further determine whether the decline in Drp1 and parkin due to CPT-mediated p53 activation occurred at a transcriptional or post-transcriptional level, we analyzed changes in mRNA levels of Drp1 and parkin. CPT treatment reduced the mRNA levels of both Drp1 and parkin in $\mathrm{p} 53^{+/+}$ neurons (Fig. 6). However, Drp1 and parkin mRNA expression were suppressed in $\mathrm{p}^{5} 3^{-1-}$ neurons as well (Fig. 6). These results suggest that the observed changes in the expression of Drp1 and parkin protein occur largely as a result of p53-dependent translational suppression and/or protein degradation since Drp1 and parkin protein levels in $\mathrm{p} 53^{-1-}$ neurons remain unchanged (Fig. $4 B, C$ ) despite their decline in mRNA levels (Fig. 6).

\section{Restoring Drp1 or parkin expression reduces CPT-induced} mitochondrial elongation and neuronal damage

CPT treatment resulted in declining Drp1 levels in a p53dependent manner and it occurred as early as $4 \mathrm{~h}$ after exposure (Fig. 4), well before commitment of cell death occurring at $8 \mathrm{~h}$ ( $50 \%$ cells committed) to $12 \mathrm{~h}$ ( $100 \%$ cells committed; (Uo et al., 2007). Parkin depletion was more protracted occurring $8-12 \mathrm{~h}$ after CPT treatment but still within the commitment window for 
apoptosis. Drp1 knockdown is neurotoxic (Uo et al., 2009), and parkin knockdown also results in mitochondrial apoptosis (Fig. 5D). Therefore, p53-dependent reduction in Drp1 and parkin levels may contribute to the loss of neuronal viability following genotoxic stress. To evaluate this possibility we overexpressed Drp1 or parkin before CPT treatment. Parkin or Drp1 overexpression significantly decreased the percentage of dying neurons $24 \mathrm{~h}$ following CPT treatment (Fig. 7A). CPT-induced elevation of activated caspase-3 expression was also significantly attenuated at this time point when neurons overexpressed Drp1 or parkin (Fig. 7B). Under these conditions, CPT treatment still suppressed Drp1 or parkin protein expression in neurons overexpressing these proteins. However, the resulting suppressed levels remained above the levels seen in control neurons (EGFPDMSO), thus maintaining the individual proteins above the physiological levels of expression even in the presence of CPT (Fig. 7B). Overexpression of Drp1 or parkin did not reciprocally prevent CPTinduced decrease of the other protein (Fig. $7 B$; the inset in the parkin blot represents an image of a longer exposure to show that Drp1 overexpression does not mitigate CPT-induced parkin loss), nor did it completely suppress CPT-induced mitochondrial apoptosis (caspase-3 activation; Fig. 7B), suggesting that the neuroprotective effects of Drp1 and parkin are not mediated by the upregulation of one protein by the other. Similarly, knockdown of Drp1 or parkin did not affect expression levels of the other (Fig. 5C,D). Overexpression of Drp1 or parkin also significantly attenuated CPT-induced mitochondrial elongation (Fig. 8). These findings suggest that the p53dependent decline in Drp1 and parkin are causally related to both the CPT-induced increase in mitochondrial length, most likely via reduced fission activity, and apoptosis.

\section{Discussion}

Neurotoxic stress associated with nervous system injury and neurodegenerative diseases induces mitochondrial dysfunction commonly coupled with mitochondrial fission (Knott et al., 2008). In the present study we determined whether genotoxic stress in postnatal cortical neurons, which activates a p53/Bax-dependent apoptotic pathway, involves changes in mitochondrial morphology. Our results demonstrate that (1) in contrast to other forms of neuronal insults, genotoxic stress did not induce mitochondrial fission, but resulted in fewer mitochondria with increased length in neurites indicative of net mitochondrial fusion; (2) genotoxic stress induced a p53dependent decline in Drp 1 and parkin expression, which occurred before the commitment point for apoptosis; and (3)
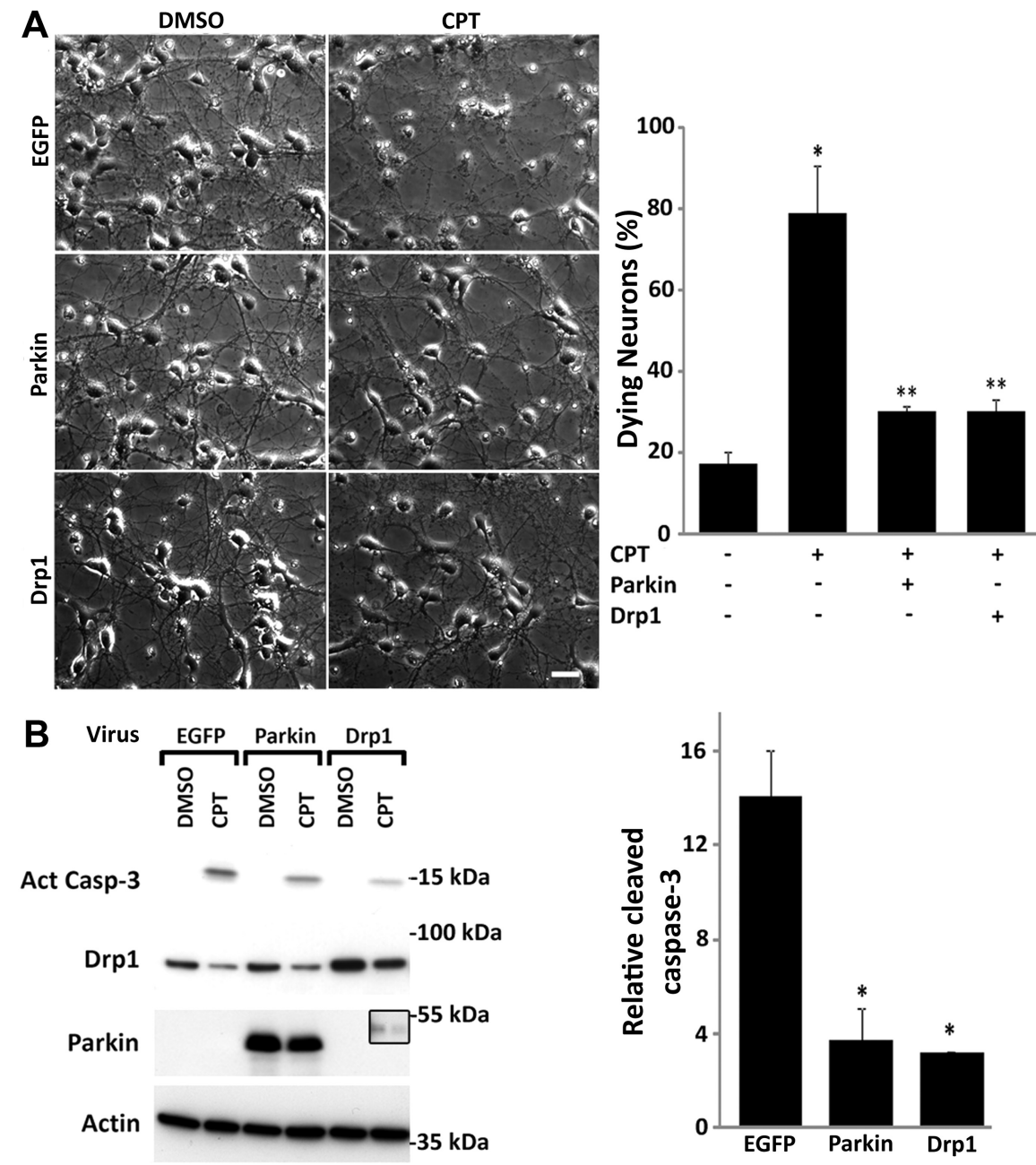

Figure 7. Increased Drp1 and parkin expression reduces (PT-induced caspase- 3 activation and cell death. $\boldsymbol{A}, \mathrm{p} 53^{+/+}$cortical neurons were infected with lentivirus (10 MOI) expressing EGFP (control virus), parkin, or Drp1 (isoform 1: Uo et al., 2009) 24 h after treatment and quantified for the percentage of dying/dead neurons. Scale bar, $25 \mu \mathrm{m}$. These data represent the mean \pm SEM two independent experiments. *, Significantly different from EGFP + DMSO ( $p<0.01$, one-way ANOVA using Tukey's post hoc test). protein samples were prepared and analyzed for expression of Drp1, parkin, and activated caspase-3 by Western blot. The inset in the parkin blot represents a prolonged exposure of the "Drp1" lanes showing that Drp1 overexpression does not prevent CPTriments. Activated caspase-3 expression was then quantified using ImageJ, normalized against actin, and presented in the different from EGFP ( $p<0.01$, one-way ANOVA using Tukey's post hoc test).

elevating Drp1 or parkin expression prevented the increase in mitochondrial length and attenuated neuronal cell death induced by genotoxic stress.

\section{Mitochondrial morphology in disease and injury: fission versus fusion}

Neurons display mitochondrial fragmentation during apoptosis induced by a wide range of stresses in vitro such as 3-NP (Liot et al., 2009), $\mathrm{H}_{2} \mathrm{O}_{2}$ (Jahani-Asl et al., 2007), nitric oxide, rotenone, and amyloid- $\beta$ peptide (Barsoum et al., 2006) and overexpression of amyloid precursor protein (Wang et al., 2008). In these studies either Drp1 inhibition or Mfn1/2 overexpression ameliorates mitochondrial fragmentation and cell death (Barsoum et al., 2006; Jahani-Asl et al., 2007; Yuan et al., 2007). Increased fission also appears to be associated with neurodegenerative conditions. Mutations in PINK1 linked to familial forms of Parkinson's dis- 

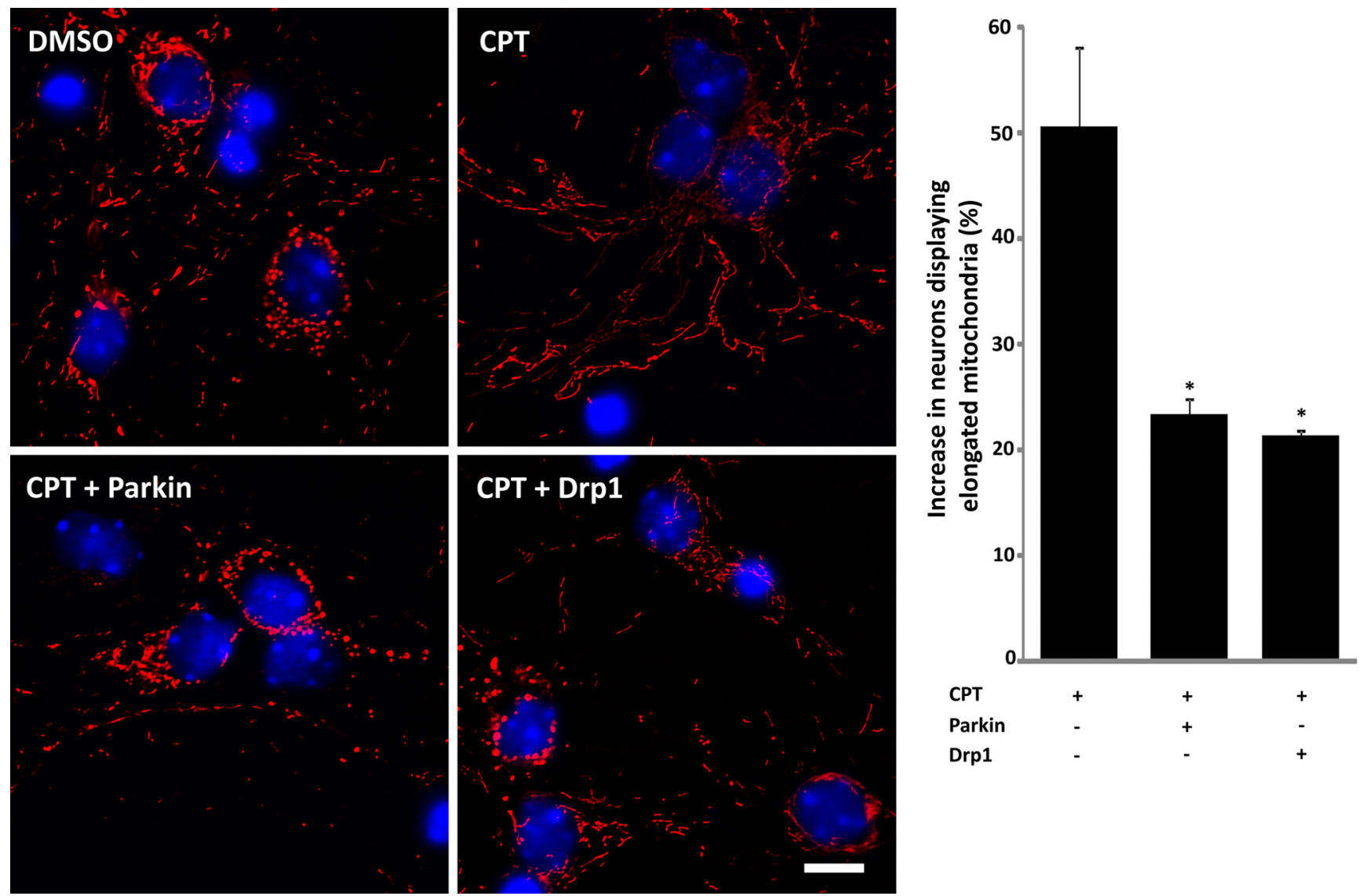

Figure 8. Increased Drp1 or parkin expression reduces CPT-induced mitochondrial elongation. $\mathrm{p} 53^{+/+}$neurons were infected with lentivirus expressing mitoDsRed2 and either parkin or wild-type Drp1 (10 MOI) $24 \mathrm{~h}$ after plating. Cells were treated with DMSO or CPT $(5 \mu \mathrm{m}) 3 \mathrm{~d}$ after infection and fixed $12 \mathrm{~h}$ after treatment. Cells were stained with Hoechst 33258 to delineate nuclei (blue) to help quantify the percentage of neurons displaying elongated mitochondria in the processes. Scale bar, $7 \mu \mathrm{m}$. The percentage of neurons displaying elongated mitochondria was quantitated as described (see Materials and Methods) and presented in the graph as the mean \pm SEM two independent experiments. *, Significantly different from CPT ( $p<0.05$, one-way ANOVA using Tukey's post hoc test).

ease are associated with mitochondrial fragmentation and impaired mitophagy in cultured neurons (Exner et al., 2007; Lutz et al., 2009; Cui et al., 2010). Affected neurons in Alzheimer's disease (Wang et al., 2009) and Huntington's disease (Kim et al., 2010) patients show progressive loss of mitochondria potentially due to increased fission. Mitochondrial fragmentation is also observed in a mouse model of stroke (Barsoum et al., 2006; Grohm et al., 2012). In marked contrast to these studies and our own observations that postnatal cortical neurons in culture exhibit fragmented mitochondria upon treatment with staurosporine, glutamate, and 3-NP, we observed elongated mitochondria in the neurites of postnatal cortical neurons treated with the DNA damaging agent, CPT. It remains to be determined how mitochondrial morphology changes in the soma where mitochondria were too dense to be defined individually. The observed response to genotoxic stress may be unique to neurons as CPT treatment of MEFs resulted in fragmented mitochondria. These results suggest that DNA damage in postnatal cortical neurons may activate pathways that affect mitochondrial morphology differently both from other acute injuries and from other cell types. Consistent with this notion was the significant reduction in Drp 1 and parkin protein levels observed in cortical neurons when treated with CPT but not with other forms of stress (Figs. 4, 5). The reduction in Drp1 and parkin levels was p53 dependent although there remains an effect of CPT on parkin levels, even in the absence of p53 (Fig. 4).
Stress-induced mitochondrial fusion is not unprecedented. SLP-2-mediated fusion occurs in response to various stresses in different cell types (Tondera et al., 2009). However, this stressinduced mitochondrial fusion is anti-apoptotic and does not involve changes in Drp1 expression (Tondera et al., 2009). In contrast, a recent finding demonstrates that in fly and neuronal models of Alzheimer's disease and related tauopathies, inappropriate expression of tau precipitates actin-mediated mislocalization of Drp1 resulting in mitochondrial elongation and neurotoxicity (DuBoff et al., 2012). The latter finding is consistent with our results demonstrating that Drp1 depletion and resulting elongated mitochondria can occur in association with apoptosis that can be ameliorated by overexpression of Drp1 (Figs. 7, 8). Also, the present study demonstrated for the first time that parkin levels in neurons decrease in response to genotoxic stress. Although these changes may not be the sole biochemical changes responsible for the observed increase in mitochondrial length, our results suggest that CPT-induced declines in the expression of Drp1 and parkin represent a molecular mechanism underlying the observed net increase in mitochondrial length because the elongated phenotype was corrected by Drp1 or parkin overexpression. This mechanism of mitochondrial elongation may be relevant to understanding some aspects of the mitochondrial toxicity associated with human neurodegenerative disease (DuBoff et al., 2012). 


\section{The mechanisms underlying the decline in Drp1 and parkin expression and activity}

Reduced expression of Drp1 is atypical of apoptosis-related changes in mitochondrial proteins. Also, reduced expression of parkin in association with apoptosis has not been reported. What potentially separates the CPT-induced neuronal cell death model from others is the involvement of p53 activation in this DNA damage-based apoptosis model. Both Drp1 and parkin genes have been shown to be transcriptionally upregulated p53 targets (Li et al., 2010; Zhang et al., 2011). These modes of transcriptional regulation demonstrated for non-neuronal cells, however, do not seem to be operational in neurons. Our results instead demonstrated p53-dependent decreases in Drp1 and parkin protein levels following DNA damage likely involving a posttranscriptional mechanism(s). CPT treatment of both $\mathrm{p} 53^{+/+}$ and $\mathrm{p} 53^{-/-}$neurons decreased mRNA levels of Drp 1 and parkin, but only $\mathrm{p} 53^{+/+}$neurons developed decreased expression of the proteins. This is consistent with the observation that p53dependent upregulation of parkin, occurring in a tissue-specific manner, is not observed in brain (Zhang et al., 2011). As such, although no direct transcriptional regulation appeared to be involved, our results involving p53 $3^{-1-}$ neurons and forced expression of p53 clearly demonstrate that declines in Drp1 and parkin in neurons occurred as a result of p53 activation (Fig. 4). Thus, p53 activation alters expression of Drp1 and parkin through cell type-specific mechanisms and, in neurons, appears to have unique implications for the regulation of the two proteins leading to genotoxic stress-induced increases in mitochondrial length. In neurons, p53 may modulate expression of unidentified molecules that alter the translation or stability of Drp1 and parkin.

Parkin is an ubiquitin E3 ligase, promoting ubiquitination of substrate proteins to mark them for proteasomal degradation. Both Drp1 (Wang et al., 2011a) and Mfn1/Mfn2 (Ziviani et al., 2010) can be targets of parkin-mediated ubiquitination and degradation. These parkin activities are unlikely to contribute to the observed CPT-induced decline in neuronal Drp1, however. The expression of parkin also declined by CPT treatment and parkin overexpression did not exacerbate the decline in Drp1 (Fig. 7B). Also, we did not observe a significant increase in Mfn1/Mfn2 in CPT-treated neurons (Uo et al., 2009), suggesting that parkin may have other targets among fusion/fission proteins in neurons.

\section{Reduced expression of Drp1/parkin mediate changes in mitochondrial morphology and apoptosis}

Our results indicate that Drp1 and parkin levels drop before neurons are committed to apoptosis and that sustained expression of Drp1 or parkin can partially rescue neurons from CPT-induced apoptosis with restoration of normal mitochondrial length. Knockdown of Drp1 (Uo et al., 2009) or parkin (Fig. 5D) expression is sufficient to compromise neuronal viability even in the absence of stress. These results collectively support the idea that the reductions in Drp1 and parkin are causally related to CPTinduced changes in mitochondrial morphology and neuronal apoptosis.

Beyond the regulation of mitochondrial morphology, the concomitant decrease in Drp1 and parkin expression may have significant functional consequences for mitophagy-mediated mitochondrial quality control. Drp1 mediates fission of dysfunctional mitochondria (Karbowski et al., 2002) while parkin marks damaged mitochondria for autophagic degradation (Narendra et al., 2008). With such functions compromised, a net increase in mitochondrial elongation may result in the unwanted preservation of dysfunctional mitochondria leading to bioenergetic defi- cits and cell death. We attempted to quantify incidences of mitochondrial structures found within autophagosomes in electron microscopic images, but there were too few autophagosomes with identifiable mitochondrial structures in either the DMSO- or CPT-treated group to assess such a possibility. These observations may alternatively suggest that parkin contributes to maintaining neuronal viability through other means aside from mitophagy. Parkin can also influence mitochondrial function by promoting mitochondrial biogenesis (Shin et al., 2011) or suppressing apoptosis via Bax ubiquitination (Johnson et al., 2012). Thus, a decline in parkin expression alone could compromise mitochondrial integrity. Furthermore, since parkin can suppress p53 expression (da Costa et al., 2009), decreasing parkin expression could result in sustained p53 expression in response to genotoxic stress. Conversely, parkin overexpression may ameliorate the CPT-induced mitochondrial phenotype by suppressing p53 expression.

\section{Concluding remarks}

Overexpression of Drp1 and parkin mitigated the observed mitochondrial phenotype and apoptosis induced by genotoxic stress. Parkin's role in neuroprotection has been previously described (Darios et al., 2003), but the neuroprotective role of Drp1 demonstrated in this study is novel contrasting with its more prevailing role in promoting apoptosis (Frank et al., 2001). Accumulated DNA damage from chronic oxidative stress is considered an important factor for the onset and progression of agerelated cognitive decline and neurodegenerative diseases (Lin and Beal, 2006; Dröge and Schipper, 2007). Neuronal regulation of the p53-dependent cellular response to stress, which is unique relative to non-neuronal cells as revealed for mitochondrial morphology in the present study, provides a clue to neuron-specific pathology manifested in many neurodegenerative diseases despite the somatic nature of individual mutations in the responsible genes. Future studies could determine whether p53-mediated suppression of Drp1 and parkin directly contribute to neuronal dysfunction through the loss of mitochondrial integrity.

\section{References}

Bae BI, Xu H, Igarashi S, Fujimuro M, Agrawal N, Taya Y, Hayward SD, Moran TH, Montell C, Ross CA, Snyder SH, Sawa A (2005) p53 Mediates cellular dysfunction and behavioral abnormalities in Huntington's disease. Neuron 47:29-41. CrossRef Medline

Barsoum MJ, Yuan H, Gerencser AA, Liot G, Kushnareva Y, Gräber S, Kovacs I, Lee WD, Waggoner J, Cui J, White AD, Bossy B, Martinou JC, Youle RJ, Lipton SA, Ellisman MH, Perkins GA, Bossy-Wetzel E (2006) Nitric oxide-induced mitochondrial fission is regulated by dynamin-related GTPases in neurons. EMBO J 25:3900-3911. CrossRef Medline

Benard G, Karbowski M (2009) Mitochondrial fusion and division: regulation and role in cell viability. Semin Cell Dev Biol 20:365-374. CrossRef Medline

Chen H, Chan DC (2005) Emerging functions of mammalian mitochondrial fusion and fission. Hum Mol Genet 14:R283-R289. CrossRef Medline

Cui M, Tang X, Christian WV, Yoon Y, Tieu K (2010) Perturbations in mitochondrial dynamics induced by human mutant PINK1 can be rescued by the mitochondrial division inhibitor mdivi-1. J Biol Chem 285: 11740-11752. CrossRef Medline

da Costa CA, Sunyach C, Giaime E, West A, Corti O, Brice A, Safe S, AbouSleiman PM, Wood NW, Takahashi H, Goldberg MS, Shen J, Checler F (2009) Transcriptional repression of $\mathrm{p} 53$ by parkin and impairment by mutations associated with autosomal recessive juvenile Parkinson's disease. Nat Cell Biol 11:1370-1375. CrossRef Medline

Dagda RK, Cherra SJ 3rd, Kulich SM, Tandon A, Park D, Chu CT (2009) Loss of PINK1 function promotes mitophagy through effects on oxidative stress and mitochondrial fission. J Biol Chem 284:13843-13855. CrossRef Medline 
Darios F, Corti O, Lücking CB, Hampe C, Muriel MP, Abbas N, Gu WJ, Hirsch EC, Rooney T, Ruberg M, Brice A (2003) Parkin prevents mitochondrial swelling and cytochrome $\mathrm{c}$ release in mitochondria-dependent cell death. Hum Mol Genet 12:517-526. CrossRef Medline

Dröge W, Schipper HM (2007) Oxidative stress and aberrant signaling in aging and cognitive decline. Aging Cell 6:361-370. CrossRef Medline

DuBoff B, Götz J, Feany MB (2012) Tau promotes neurodegeneration via DRP1 mislocalization in vivo. Neuron 75:618-632. CrossRef Medline

Exner N, Treske B, Paquet D, Holmström K, Schiesling C, Gispert S, CarballoCarbajal I, Berg D, Hoepken HH, Gasser T, Krüger R, Winklhofer KF, Vogel F, Reichert AS, Auburger G, Kahle PJ, Schmid B, Haass C (2007) Loss-of-function of human PINK1 results in mitochondrial pathology and can be rescued by parkin. J Neurosci 27:12413-12418. CrossRef Medline

Frank S, Gaume B, Bergmann-Leitner ES, Leitner WW, Robert EG, Catez F, Smith CL, Youle RJ (2001) The role of dynamin-related protein 1, a mediator of mitochondrial fission, in apoptosis. Dev Cell 1:515-525. CrossRef Medline

Grohm J, Kim SW, Mamrak U, Tobaben S, Cassidy-Stone A, Nunnari J, Plesnila N, Culmsee C (2012) Inhibition of Drp1 provides neuroprotection in vitro and in vivo. Cell Death Differ 19:1446-1458. CrossRef Medline

Jahani-Asl A, Cheung EC, Neuspiel M, MacLaurin JG, Fortin A, Park DS, McBride HM, Slack RS (2007) Mitofusin 2 protects cerebellar granule neurons against injury-induced cell death. J Biol Chem 282:23788-23798. CrossRef Medline

Johnson BN, Berger AK, Cortese GP, Lavoie MJ (2012) The ubiquitin E3 ligase parkin regulates the proapoptotic function of Bax. Proc Natl Acad Sci U S A 109:6283-6288. CrossRef Medline

Karbowski M, Lee YJ, Gaume B, Jeong SY, Frank S, Nechushtan A, Santel A, Fuller M, Smith CL, Youle RJ (2002) Spatial and temporal association of Bax with mitochondrial fission sites, Drp1, and Mfn2 during apoptosis. J Cell Biol 159:931-938. CrossRef Medline

Karbowski M, Norris KL, Cleland MM, Jeong SY, Youle RJ (2006) Role of Bax and Bak in mitochondrial morphogenesis. Nature 443:658-662. CrossRef Medline

Kim J, Moody JP, Edgerly CK, Bordiuk OL, Cormier K, Smith K, Beal MF, Ferrante RJ (2010) Mitochondrial loss, dysfunction and altered dynamics in Huntington's disease. Hum Mol Genet 19:3919-3935. CrossRef Medline

Kim KY, Stevens MV, Akter MH, Rusk SE, Huang RJ, Cohen A, Noguchi A, Springer D, Bocharov AV, Eggerman TL, Suen DF, Youle RJ, Amar M, Remaley AT, Sack MN (2011) Parkin is a lipid-responsive regulator of fat uptake in mice and mutant human cells. J Clin Invest 121:3701-3712. CrossRef Medline

Knott AB, Perkins G, Schwarzenbacher R, Bossy-Wetzel E (2008) Mitochondrial fragmentation in neurodegeneration. Nat Rev Neurosci 9:505518. CrossRef Medline

Li J, Donath S, Li Y, Qin D, Prabhakar BS, Li P (2010) miR-30 regulates mitochondrial fission through targeting p53 and the dynamin-related protein-1 pathway. PLoS Genet 6:e1000795. CrossRef Medline

Lin MT, Beal MF (2006) Mitochondrial dysfunction and oxidative stress in neurodegenerative diseases. Nature 443:787-795. CrossRef Medline

Liot G, Bossy B, Lubitz S, Kushnareva Y, Sejbuk N, Bossy-Wetzel E (2009) Complex II inhibition by 3-NP causes mitochondrial fragmentation and neuronal cell death via an NMDA- and ROS-dependent pathway. Cell Death Differ 16:899-909. CrossRef Medline

Lutz AK, Exner N, Fett ME, Schlehe JS, Kloos K, Lämmermann K, Brunner B, Kurz-Drexler A, Vogel F, Reichert AS, Bouman L, Vogt-Weisenhorn D, Wurst W, Tatzelt J, Haass C, Winklhofer KF (2009) Loss of parkin or PINK1 function increases Drp1-dependent mitochondrial fragmentation. J Biol Chem 284:22938-22951. CrossRef Medline

Messmer U, Brüne B (1996) Nitric oxide-induced apoptosis: p53dependent and p53-independent signalling pathways. Biochem J 319 299-305.

Morrison RS, Kinoshita Y, Johnson MD, Guo W, Garden GA (2003) p53Dependent cell death signaling in neurons. Neurochem Res 28:15-27. CrossRef Medline
Narendra D, Tanaka A, Suen DF, Youle RJ (2008) Parkin is recruited selectively to impaired mitochondria and promotes their autophagy. J Cell Biol 183:795-803. CrossRef Medline

Poole AC, Thomas RE, Andrews LA, McBride HM, Whitworth AJ, Pallanck LJ (2008) The PINK1/Parkin pathway regulates mitochondrial morphology. Proc Natl Acad Sci U S A 105:1638-1643. CrossRef Medline

Shin JH, Ko HS, Kang H, Lee Y, Lee YI, Pletinkova O, Troconso JC, Dawson VL, Dawson TM (2011) Paris (ZNF746) repression of PGC- $1 \alpha$ contributes to neurodegeneration in Parkinson's disease. Cell 144:689-702. CrossRef Medline

Tomicic MT, Christmann M, Kaina B (2005) Topotecan-triggered degradation of topoisomerase $\mathrm{i}$ is p53-dependent and impacts cell survival. Cancer Res 65:8920-8926. CrossRef Medline

Tondera D, Grandemange S, Jourdain A, Karbowski M, Mattenberger Y, Herzig S, Da Cruz S, Clerc P, Raschke I, Merkwirth C, Ehses S, Krause F, Chan DC, Alexander C, Bauer C, Youle R, Langer T, Martinou JC (2009) SLP-2 is required for stress-induced mitochondrial hyperfusion. EMBO J 28:1589-1600. CrossRef Medline

Uo T, Kinoshita Y, Morrison RS (2007) Apoptotic actions of p53 require transcriptional activation of PUMA and do not involve a direct mitochondrial/cytoplasmic site of action in postnatal cortical neurons. J Neurosci 27:12198-12210. CrossRef Medline

Uo T, Dworzak J, Kinoshita C, Inman DM, Kinoshita Y, Horner PJ, Morrison RS (2009) Drp1 levels constitutively regulate mitochondrial dynamics and cell survival in cortical neurons. Exp Neurol 218:274-285. CrossRef Medline

Wang H, Song P, Du L, Tian W, Yue W, Liu M, Li D, Wang B, Zhu Y, Cao C, Zhou J, Chen Q (2011a) Parkin ubiquitinates Drp1 for proteasomedependent degradation. J Biol Chem 286:11649-11658. CrossRef Medline

Wang X, Su B, Siedlak SL, Moreira PI, Fujioka H, Wang Y, Casadesus G, Zhu $\mathrm{X}$ (2008) Amyloid- $\beta$ overproduction causes abnormal mitochondrial dynamics via differential modulation of mitochondrial fission/fusion proteins. Proc Natl Acad Sci U S A 105:19318-19323. CrossRef Medline

Wang X, Su B, Lee HG, Li X, Perry G, Smith MA, Zhu X (2009) Impaired balance of mitochondrial fission and fusion in Alzheimer's disease. J Neurosci 29:9090-9103. CrossRef Medline

Wang X, Winter D, Ashrafi G, Schlehe J, Wong YL, Selkoe D, Rice S, Steen J, LaVoie MJ, Schwarz TL (2011b) PINK1 and parkin target miro for phosphorylation and degradation to arrest mitochondrial motility. Cell 147:893-906. CrossRef Medline

Wu C-Y, Gómez-Curet I, Funanage VL, Scavina M, Wang W (2009) Increased susceptibility of spinal muscular atrophy fibroblasts to camptothecin is p53-independent. BMC Cell Biology 10:40. CrossRef Medline

Xiang H, Hochman DW, Saya H, Fujiwara T, Schwartzkroin PA, Morrison RS (1996) Evidence for p53-mediated modulation of neuronal viability. J Neurosci 16:6753-6765. Medline

Xiang H, Kinoshita Y, Knudson CM, Korsmeyer SJ, Schwartzkroin PA, Morrison RS (1998) Bax involvement in p53-mediated neuronal cell death. J Neurosci 18:1363-1373. Medline

Youle RJ, Karbowski M (2005) Mitochondrial fission in apoptosis. Nat Rev Mol Cell Biol 6:657-663. Medline

Young KW, Piñon LG, Bampton ET, Nicotera P (2010) Different pathways lead to mitochondrial fragmentation during apoptotic and excitotoxic cell death in primary neurons. J Biochem Mol Toxicol 24:335-341. CrossRef Medline

Yuan H, Gerencser AA, Liot G, Lipton SA, Ellisman M, Perkins GA, BossyWetzel E (2007) Mitochondrial fission is an upstream and required event for bax foci formation in response to nitric oxide in cortical neurons. Cell Death Differ 14:462-471. CrossRef Medline

Zhang C, Lin M, Wu R, Wang X, Yang B, Levine AJ, Hu W, Feng Z (2011) Parkin, a p53 target gene, mediates the role of p53 in glucose metabolism and the Warburg effect. Proc Natl Acad Sci U S A 108:16259-16264. CrossRef Medline

Ziviani E, Tao RN, Whitworth AJ (2010) Drosophila Parkin requires PINK1 for mitochondrial translocation and ubiquitinates Mitofusin. Proc Natl Acad Sci U S A 107:5018-5023. CrossRef Medline 